\title{
Novel transvenous catheter mapping and ablation could supersede invasive antiepileptic surgery
}

Catheter-guided mapping and electrical ablation are commonly used to treat cardiac arrhythmias, and these techniques may soon be available to treat patients with epilepsy. New research from investigators at the Mayo Clinic in Rochester, MN, demonstrates the feasibility and efficacy of this approach to locate and ablate seizure foci in nonhuman primates.

\section{4 ...we ... envision a time when epilepsy is treated much the same way as cardiac ... disturbances... 77}

Current treatment of epilepsy is limited to failure-prone pharmacological therapy or effective but highly invasive surgical approaches. With the aim of providing a minimally invasive therapeutic option, Samuel Asirvatham and colleagues are developing a catheter ablation technique for use in the brain.
In an earlier study, the researchers had demonstrated the feasibility of this technology in pigs. "We were able to record electrical signals and also ... to deliver radiofrequency energy to produce fairly predictable lesions in what would be targeted sites in the brain," explains Asirvatham. "We then moved to a nonhuman primate model, since this was the only viable option to mimic the venous system and anatomy of humans to further demonstrate quantitative safety and efficacy of this approach."

The team used soft, saline-cooled, over-wire catheters that were much smaller than those used for cardiac arrhythmia ablation. Using these catheters, the researchers were able to navigate the venous system and access deep areas of the brain without causing venous lacerations, or haemorrhagic or embolic complications.

In 11 baboons and three dogs, the investigators successfully mapped brain activity by guiding the catheter through the cerebral venous system. Seizures were induced in the animals by high-frequency pacing or intracortical penicillin injection, and the presumed seizure focus was subsequently ablated by use of radiofrequency energy without the need for extensive manipulation.

"Instead of the need for either surgery or invasive mapping to identify the site that needs to be surgically improved, we can perhaps envision a time when epilepsy is treated much the same way as cardiac electrical disturbances with a single day of hospital stay and a nonsurgical approach," Asirvatham hopes.

Clemens Thoma

Original Article Henz, B. D. et al. Advances in
radiofrequency ablation of the cerebral cortex in primates
using the venous system: improvements for treating
epilepsy with catheter ablation technology. Epilepsy Res.
doi:10.1016/j.eplepsyres.2014.04.002

Original Article Henz, B. D. et al. Advances in radiofrequency ablation of the cerebral cortex in primates doi:10.1016/j.eplepsyres.2014.04.002 